\title{
Recuperación de un ecosistema perturbado en el cerro El Castillo del Bosque Protector Mindo Nambillo
}

\section{Recovery of the ecosystem disturbed in El Castillo hill of the protected forest Mindo Nambillo}

\author{
Herman Hernández Benalcázar ${ }^{1}$ \\ ${ }^{1}$ Universidad Central del Ecuador. Facultad de Ciencias Agricolas. Carrera de Turismo Ecológico. \\ Jerónimo Leiton y Av. La Gasca s/n. Ciudadela Universitaria. Quito. 170521. Ecuador.
}

\begin{abstract}
Resumen
En el cerro El Castillo del Bosque Protector Mindo Nambillo, por donde atraviesa la ruta del Oleoducto de Crudos Pesados (OCP), se realizó la presente investigación descriptiva. Es una zona de vida Bosque Muy Húmedo Montano, también considerado como Bosque de Neblina Montano. En la construcción del Oleoducto, en todo su recorrido y en un ancho de $15 \mathrm{~m}$, se provocaron fuertes perturbaciones antrópicas. Después de tres meses de provocada la perturbación, y de haberse realizado trabajos de recomposición de la vegetación en la zona, se hicieron observaciones de la cubierta vegetal para verificar la recuperación del ecosistema del área, comparando los resultados obtenidos con la descripción de la línea base presentada por ENTRIX en el 2001. El método del Área Mínima se utilizó para el conteo y análisis de la cubierta vegetal, en una superficie de $64 \mathrm{~m}^{2}$, donde se encontraron 93 especies de plantas herbáceas y arbustivas, de 29 familias. Ninguna de las especies herbáceas encontradas fueron incluidas en el inventario realizado en la Línea Base de la OCP antes de la perturbación. Al hacer la comparación entre las plantas herbáceas que se encontró en el lugar abierto con el interior del bosque tampoco se las encontraron, con lo que se comprueba que en la recuperación y recomposición de un ecosistema intervienen especies pioneras herbáceas y arbustivas que migraron a través de muchos factores o que sus semillas se mantienen en latencia por mucho tiempo y de esa manera un ecosistema se regenera, se recupera y vuelve a su estado de equilibrio, mientras no existan otras perturbaciones.
\end{abstract}

Palabras clave: ecosistemas perturbados, regeneración natural, perturbaciones antrópica.

\begin{abstract}
This research was carried out in El Castillo Hill of the Protected Forest Mindo Nambillo, where the heavy crude oil pipeline (OCP) route crosses. It is a life zone known as humid montane forest, also considered as Cloud Forest Montano. Along the construction of the pipeline, and $15 \mathrm{~m}$ wide, a strong human disturbance was caused. After three months of the caused disturbance and the rebuilding of vegetation, a study of the vegetation cover for verifying the ecosystem recovery was performed. The results were compared with the description of the baseline made by ENTRIX in 2001. Minimal Area Method was used, with $64 \mathrm{~m}^{2}$ plot size. As result, 93 species of herbaceous and woody plants, of 29 families, were found. None of the herbaceous species found in the study were observed at the inventory of the OCP baseline. Moreover, no coincidences of herbaceous plants where found between open area and interior forest. So, intervention of herbaceous pioneer species and shrubs, which migrate through several factors or which seeds latency is long-term, is probed in the recovery and recomposition of an ecosystem, and this the way how an ecosystem regenerates, recovers backs to its equilibrium state, while no other disturbances exist.
\end{abstract}

Keywords: disturbed ecosystems, natural regeneration, anthropogenic disturbances. 


\section{Introducción}

En la construcción de grandes y pequeñas obras de infraestructura se producen perturbaciones que provocan desequilibrios en los ecosistemas. Estas perturbaciones son de dos tipos: naturales y antrópicas. En los dos casos, la naturaleza busca equilibrarse en corto, mediano o largo tiempo, siempre y cuando las perturbaciones no sean permanentes o se provoque con exagerada frecuencia. Las perturbaciones antrópicas son las que más provocan desequilibrios pues producen impactos de consideración que llegan incluso a dañar los ciclos naturales, al punto de desaparecer una o varias especies, Sin embargo, el hombre puede ser uno de los mejores agentes de dispersión que ayuden al establecimiento de muchas especies que tienen dificultades de establecerse a fin de lograr la inmediata recuperación del sistema (Hernández, 1996).

Varios estudios han demostrado que solo cuando se efectúan grandes aberturas de un bosque o se realicen parches en un espacio abierto, es posible la pérdida gradual de especies, después de la subdivisión del hábitat, pero también es posible ganar otras especies mientras ocurre el transcurso normal de la sucesión (Robinson et al., 1992). En una primera etapa, apenas las especies con semillas pequeñas pueden establecerse en áreas degradadas. A medida que la vegetación forestal se restablece en esas áreas, nuevos elementos del bosque deberán entrar como participantes del proceso (Uhl et al., 1991), es el caso de los animales, desde los más pequeños hasta los más grandes y complejos. Por último, dentro del proceso de sucesión es muy importante considerar el aspecto de competencia y asociaciones simbióticas que son mecanismos adaptados por muchas especies para sobrevivir (Finegan, 1992).

En primera instancia, las plantas que dominan después de una perturbación intensiva son las herbáceas, especialmente las gramíneas. Así lo demuestra un trabajo de investigación realizado por Junk \& Piedade (1993) en las riberas del río Amazonas, cerca de la ciudad de Manaos.

Aunque resulta bastante difícil describir el arribo, el establecimiento y las relaciones de asociación y competencia entre especies, dentro del modelo de sucesión de Connell \& Slatyer (1977) se trata de explicar las relaciones entre las diferentes especies que llegan a poblar un determinado lugar de una perturbación (McCook, 1994).
Junk \& Piedade (1993) encontraron algunos atributos en las especies herbáceas que dominaron los terrenos perturbados: 1 . Resistencia de las semillas y esporas a las inundaciones y la desecación, 2 . Cortos ciclos reproductivos, 3. Altos índices de reproducción, 4. Alta producción primaria, 5. Tolerancia a las plantas adultas, a las inundaciones y sequías y, 6. Adaptaciones para mantenerse con diferentes niveles de agua. El corto ciclo de vida y el alto índice reproductivo, permiten una rápida colonización del hábitat perturbado y la sucesión de una población estratégica perdida (Junk \& Piedade, 1993).

Después de una perturbación en un ecosistema el paisaje se transforma; se produce de estado en estado una sucesión de formaciones vegetales arbustivas por la reconstrucción del ecosistema característico de la zona concerniente (Anderson, 1990). Se trata de una evolución ordenada y previsible, verdadero desarrollo del ecosistema terminal. La formación terminal de estructura y de composición florística estables dentro de las condiciones definidas por el clima regional, es el clímax (Barbault, 1990). Para Odum (1969), sucesión ecológica es el proceso ordenado de desarrollo de las comunidades, efectuándose según una dirección bastante constante y por tanto previsible (Daget \& Gordon, 1970). Sobre la base de estos conceptos, puede decirse que la sucesión secundaria es la respuesta natural a una perturbación. En consecuencia, para hablar de sucesión secundaria, es necesario hablar de las perturbaciones (Barbault, 1990).

En pastizales abandonados sujetos a poco uso se observó regeneración forestal vigorosa. La media de acumulación de la biomasa a nivel del suelo es de $10 \mathrm{t} \mathrm{ha}^{-1}$ año ${ }^{-1} \mathrm{u} 80 \mathrm{t}$ después de 8 años (a groso modo o tercio de los niveles de biomasa de bosques maduros), se encontraron retoños de 20 árboles por cada $100 \mathrm{~m}^{2}$. En pastizales abandonados sujetos a un moderado uso fueron también desarrollando características del bosque, pero la acumulación de la biomasa fue solamente de $5 \mathrm{t} \mathrm{ha}^{-1} \mathrm{año}^{-1}$ (la mitad del índice presentado en pastizales de poco uso). Individuos alcanzan la vía del brote, de semilla a bancos de semillas, o semillas dispersas, pero la riqueza de tres especies fue solamente más baja que en sitios de poco uso y el número de árboles de bosque fue menor. Finalmente, en pastizales abandonados, sujetos al mayor uso, tuvieron al menos distintos patrones de sucesión. Los sitios solo de 8 años fueron dominados por poaceas y asperezas, 
con apenas un árbol por $100 \mathrm{~m}^{2} \mathrm{y}$ una acumulación de biomasa a nivel del suelo de $0.6 \mathrm{t} \mathrm{ha}^{-1} \mathrm{año}^{-1}$, un valor solamente alrededor de $6 \%$ de lo encontrado en sitios de poco uso (Uhl et al., 1990).

Después de una perturbación donde existe remoción del suelo, una gran parte de las raíces de la mayoría de las plantas están en los horizontes altos del suelo. Generalmente del 65 al 80\% de las raíces se encuentran sobre las 6 pulgadas. El volumen total de las raíces decrece como la profundidad del suelo se incrementa (USDA, 1988). Dentro de sus galerías las lombrices de tierra ingieren un volumen importante de suelo que transportan, aflojándolo en el suelo o en la superficie. La masa de desecho de las lombrices ha sido evaluada en $300 \mathrm{t} \mathrm{ha}^{-1} \mathrm{año}^{-1}$ en las zonas temperadas. En las zonas tropicales, donde la temperatura elevada incrementa la actividad de las lombrices de tierra, esta masa se estima entre 850 - $1150 \mathrm{t} \mathrm{ha}^{-1} \mathrm{año}^{-1}$, de las cuales $25 \mathrm{t}$ serían depositadas en la superficie. También las lombrices pueden ingerir 10 veces su propia masa de suelo por día, y hasta 36 veces en el caso de lombrices jóvenes (Motalib \& Rida, 1994).

Cuando existen alteraciones, como el levantamiento de la capa vegetal, el movimiento del suelo por actividad agrícola u otras actividades, se producen efectos de desequilibrio en el sistema, porque se expone a los elementos constituyentes del mismo a otras condiciones, sean estas de luz, de temperatura, de aire y al golpe directo del agua cuando llueve, sin embargo, también es un sistema que se recupera inmediatamente, según la intensidad de la alteración o perturbación.

\section{Materiales y métodos}

El sitio de estudio fue el cerro El Castillo del Bosque Protector Mindo Nambillo, localizado en las coordenadas $00^{\circ} 02^{\prime} 52.0^{\prime \prime} \mathrm{S}$; $78^{\circ} 38^{\prime} 48.9^{\prime \prime}$ W y 2.625 m.s.n.m. Es un bosque muy húmedo de una zona nublada, es primario intervenido, bosque perenne, corresponde a la región bioclimática húmeda subtropical y zona de vegetación de bosque muy húmedo montano según la clasificación de zonas de vida de Holdridge (BmhM) (Cañadas Cruz, 1983).

Es una zona con pendientes muy pronunciadas, se observa roca meteorizada, suelo bien drenado y fácilmente deleznable si no se mantiene una cubierta vegetal.

En este lugar se realizaron obras de ingeniería a fin de colocar la tubería del Oleoducto de Crudos Pesados (OCP), ejecutar aseguramientos y sostenimiento de la misma, Esta actividad antrópica provocó un gran impacto en el ecosistema, específicamente en la flora y la fauna del lugar (Figura 1).

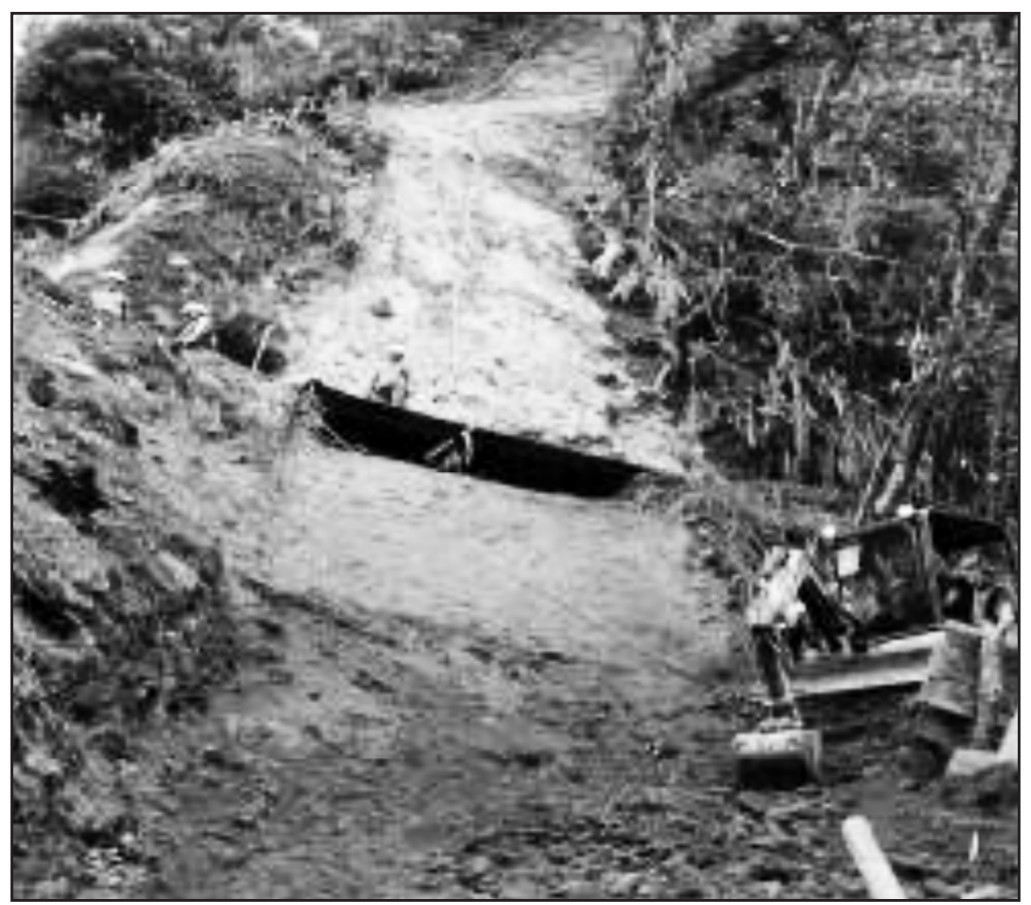

Figura 1. Obra de ingeniería en fase de construcción. 
En una primera visita se hizo el reconocimiento del área. Se recorrió desde el cerro Guarumos, pasando por los cerros El Castillo y el Campanario, hasta llegar al campamento de Bella vista. En este recorrido se determinó el lugar donde se debían hacer las observaciones y se tomaron algunos datos de referencia.

El lugar determinado para realizar las observaciones fue el cerro El Castillo, tanto en la parte oriental del cerro como en la parte occidental, según las siguientes consideraciones: a) Se observa que en este lugar es donde se hizo una obra de ingeniería de gran envergadura para la ubicación de la tubería, b) Es un lugar bastante frágil, por encontrarse en la cresta de montaña, c) Por el hecho de haber sido un lugar difícil de trabajar para realizar las obras de ingeniería, ya sea por la remoción de tierra, la co- locación de la tubería, el aseguramiento y el sostenimiento de la misma, debió haberse provocado un gran impacto en el ecosistema, específicamente en la flora y la fauna del lugar, d) Porque se observa que para evitar escurrimientos de tierra hacia las laderas adyacentes, se utilizaron materiales de hormigón y geo-membranas de sostenimiento.

En una segunda visita se realizó el conteo de las especies herbáceas dentro del Derecho de Vía, utilizando el método del Área Mínima (Barbour, Burk \& Pitts, 1973) a fin de hacer el análisis cuantitativo de la flora que se ha restablecido después de la perturbación producida, incluyendo las tres especies sembradas Phaseolus polyanthus (gualea), Paspalum sp. (nudillo) y Panicum polygunatum (cola de conejo), por recomendación de la Fundación Jatun Sacha (Figura 2).

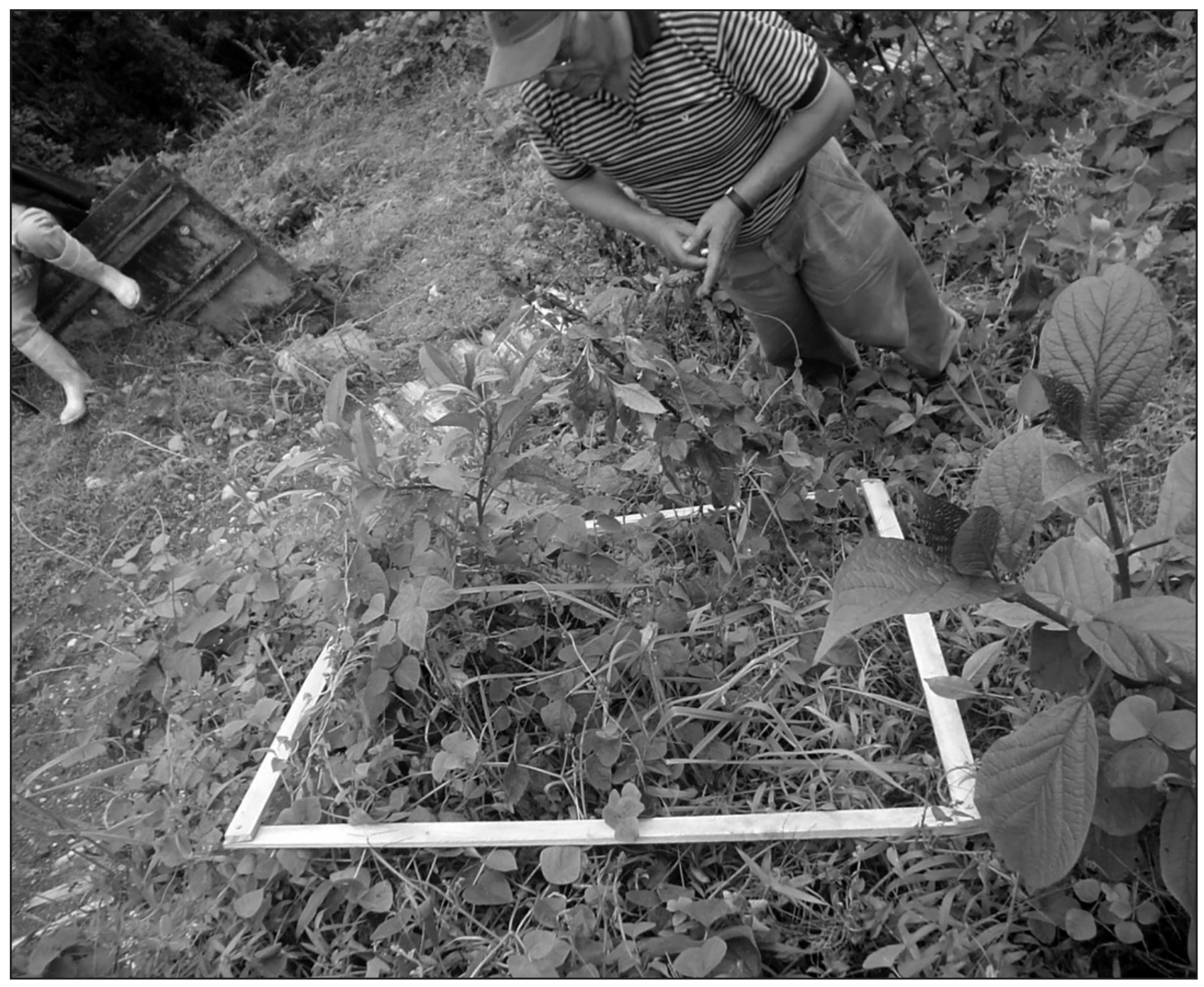

Figura 2. Área Mínima, conteo e identificación. 
Finalmente, se hicieron las comparaciones entre las especies herbáceas existentes en el interior del bosque y la Línea Base realizada por la empresa ENTRIX, en el 2001 (OCP Ecuador S.A., 2001) para la OCP, con los resultados obtenidos en este trabajo.

\section{Resultados}

El sitio de estudio es un bosque intervenido porque tiempo atrás fue explotado, según referencias de moradores que manifiestan que se extrajo madera, especialmente de cedro, y según se recoge en el Plan General de Desarrollo de Pichincha (Gobierno de la Provincia de Pichincha, 2002), se han encontrado señales de la presencia de cedros en el bosque protector Mindo Nambillo. Esto se confirmó al encontrar algunos ejemplares de esta especie maderable, al igual que de encino.

En la lista de especies de plantas inventariadas por la empresa ENTRIX para la OCP en el 2001 (Línea base), no se encuentra ninguna de las plantas herbáceas encontradas en el área observada, ni se encuentran modificaciones de los registros de monitoreo desde el primero hasta el séptimo realizado en el 2005 (OCP Ecuador S.A., 2005).

En el interior del bosque tampoco se encuentran las especies herbáceas que corresponden a las familias enlistadas en la Tabla 1. Sin embargo, en el margen de la perturbación se encontraron las especies: Carludovica palmata (Cyclantaceae), Gusmania sp. (Bromeliaceae), Zagalita sp. (Ericaceae), Palicuria sp (Rubiaceae), a las que se las puede considerar que comparten los dos ambientes.

El área mínima en la que se encuentran casi todas las especies es de $64 \mathrm{~m}^{2}$ (Figura 3). Cabe señalar que la mayoría de especies encontradas y contadas son herbáceas y muy pocas arbustivas. En el área mínima de $64 \mathrm{~m}^{2}$ se encontraron 93 especies en 29 familias, distribuidas según se muestra en la Tabla 1. La mayoría de especies que se encuentran en el lugar, son especies pioneras y heliófilas, de las cuales el 18\% corresponde a la familia de las asteráceas y el $10 \%$ a las poáceas. Pocas de estas especies pioneras se encuentran en el interior del bosque.

$\mathrm{N}^{\circ}$ acumulado de especies en el área mínima de $64 \mathrm{~m} 2$

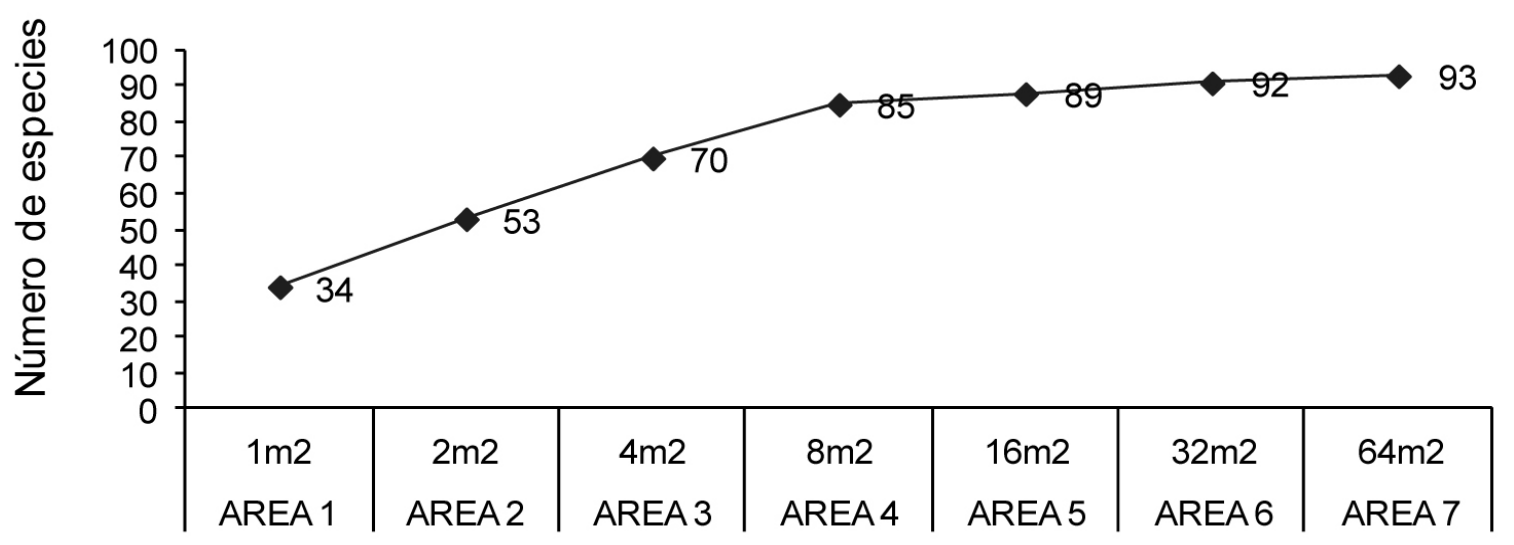

Figura 3. Número de especies encontradas en el Área Mínima. 
Tabla 1. Número de familias y especies vegetales encontradas

\begin{tabular}{|c|c|c|}
\hline $\mathbf{N}^{\circ}$ de Orden & Familias & $\mathbf{N}^{\circ}$ de Especies \\
\hline 1 & Fabaceae & 4 \\
\hline 2 & Poaceae & 9 \\
\hline 3 & Asteraceae & 17 \\
\hline 4 & Commelinacea & 2 \\
\hline 5 & Oxalidaceae & 3 \\
\hline 6 & Malvaceae & 4 \\
\hline 7 & Helecho & 6 \\
\hline 8 & Musgos & 3 \\
\hline 9 & Hongos & 1 \\
\hline 10 & Poligalaceae & 3 \\
\hline 11 & Hapatica & 1 \\
\hline 12 & Ciperaceae & 2 \\
\hline 13 & Apiaceae & 2 \\
\hline 14 & Ericaceae & 3 \\
\hline 15 & Gesneriaceae & 2 \\
\hline 16 & Equicetaceae & 1 \\
\hline 17 & Meliaceae & 4 \\
\hline 18 & Solanaceae & 3 \\
\hline 19 & Plantaginaceae & 1 \\
\hline 20 & Poligonaceae & 3 \\
\hline 21 & Gunneraceae & 2 \\
\hline 22 & Araceae & 1 \\
\hline 23 & Verbenacea & 3 \\
\hline 24 & Phitolacaceae & 1 \\
\hline 25 & Urticaceae & 2 \\
\hline 27 & Bromeliaceae & 7 \\
\hline 28 & Arrayan & 1 \\
\hline 29 & Pumamaqui & 1 \\
\hline 29 & Yaloman & 1 \\
\hline
\end{tabular}

\section{Discusión}

En el caso de estudio, la perturbación fue provocada, intensa, temporal de una sola vez y de corta duración, lo cual provocó la desaparición de varios individuos de árboles, arbustos y hierbas, y la huida de muchos animales. Pero, se considera muy difícil que esta perturbación haya provocado la desaparición de especies animales o vegetales. Por otra parte, la abertura del bosque en el Derecho de Vía (DDV), para la construcción del oleoducto, llega al $1.5 \%$ por km².
Según se observa, es indudable que existió un gran impacto negativo para la flora, la fauna y el paisaje en la etapa de construcción, especialmente en el cerro El Castillo, por el hecho de que hubo que talar los árboles en el DDV, levantar la capa vegetal y remover la tierra para enterrar el tubo. Además, se produjo por la presencia de un buen número de personas que debían realizar los trabajos, la transportación de los tubos y demás materiales, y el ruido que se produce en este tipo de obras. 


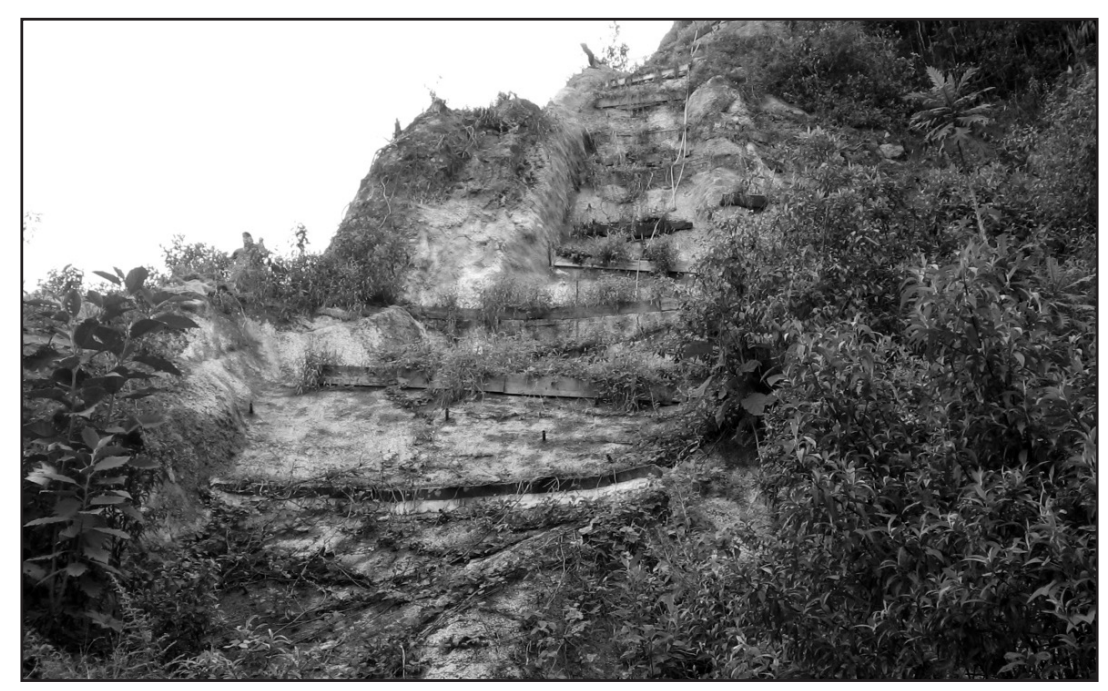

Figura 4. Fase de mantenimiento del oleoducto.

En el conteo de las especies herbáceas dentro del DDV, se observó que la flora en el sitio perturbado se había restablecido. Entre las especies de plantas encontradas se incluyen las tres especies sembradas Phaseolus polyanthus (gualea), Paspalum sp. (nudillo) y Panicum polygunatum (cola de conejo). Las especies que se encuentran con más frecuencia son aquellas que corresponden a la familia de las Asteraceae, seguido de las Poaceae y luego las pteridofitas, representadas por los helechos.
A pesar de que existió un gran impacto negativo para la flora y la fauna, después de tres meses de haber concluido la obra de ingeniería se observó que la vegetación se ha restablecido con gran vigor, porque el número de especies herbáceas encontradas en los espacios abiertos es mayor que las encontradas en el interior del bosque (Figuras 4 y 5).

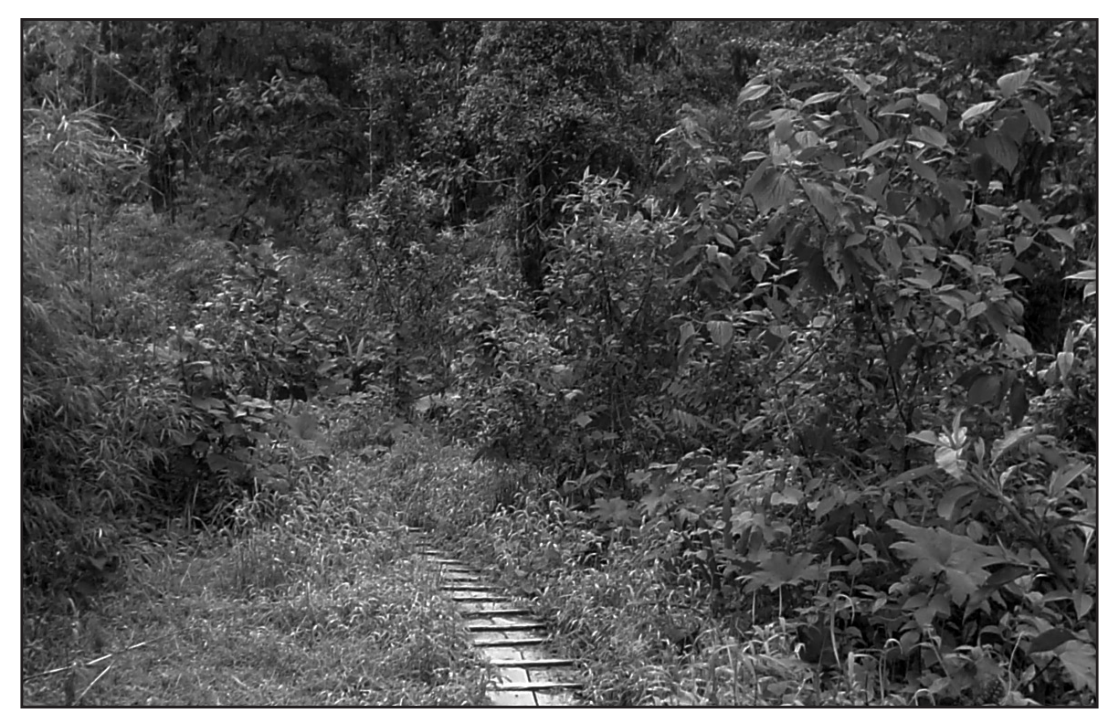

Figura 5. Respuesta de la vegetación, después de la perturbación.

\section{Conclusiones}

Por el hecho de que la perturbación fue temporal y de un tiempo corto, además, por no existir ninguna otra intervención a más de los trabajos de mantenimiento de la vía, puede considerarse como un barbecho abandonado, lo cual ha permitido que la naturaleza recupere su equilibrio y se restablezca el ecosistema. Prueba de ello 
es que en esta área, que fue bastante afectada, se han encontrado 93 especies de plantas herbáceas y arbustivas, en 29 familias. Estas especies no se encuentran registradas en el inventario de la Línea Base realizado en el 2001, ni se encuentran modificaciones de los re-

\section{Referencias}

Anderson, A. (1990). Extraction and forest management by rural inhabitants in the Amazon Estuary. En Anderson, A. (ed.) Alternatives to deforestation; Steps toward sustainable use of the Amazon rain forest. Columbia University, Press New York, pp. 65-85.

Barbault, R. (1990). Écologie générale Structure et fonctionnement de la bosphére. $2^{\circ}$ édition. Ed. Masson, Paris.

Barbour, M.G., Burk, J.H. \& Pitts, W.D: (1973). Terrestrial Plant Ecology. The Benjamín / Cummings publishing Company, Inc. Menlo Park, California. Second Edition.

Cañadas Cruz, L. (1983). El mapa bioclimático y ecológico del Ecuador. MAG, PRONAREG. Quito, Ecuador

Connell, J.H. \& Slatyer, R.O. (1977). Mechanisms of succession in natural communities and their role in community stability and organization. The American Naturalist, 111(982), 1119-1144.

Daget, P. \& Gordon, M. (1970). Vocabulaire D'Écologie. Seconde édition.

Finegan, B. (1992). Bases ecológicas para la silvicultura y Curso intensivo internacional de silvicultura y manejo de bosques naturales tropicales, Turrialba, Costa Rica. Centro Agronómico Tropical de Investigación y Enseñanza, CATIE.

Gobierno de la Provincia de Pichincha (2002). Plan General de desarrollo de la Provincia de Pichincha, Diagnostico social. Tomo 1, abril 2002.

Hernández, H. (1996). Crecimiento y producción iniciales de 15 especies de árboles tropicales de la Amazonía ecuatoriana de estados sucesionales diferentes. Memoire presenté comme exigence partielle de la Maitrese en Biologie.

Junk, J. \& Piedade, T. (1993). Herbaceus plants of the Amazon flooplain near Manaus: Species di gistros de monitoreo desde el primero hasta el séptimo realizado en el 2005. Estos resultados demuestran que existe una recuperación vegetal muy satisfactoria en el área intervenida con especies nuevas que migraron o permanecieron en latencia por mucho tiempo.

versity and adaptation to the flood pulse. Amazonian, XII (3/4), 467-484.

McCook, L. (1994). Understanding ecological community succession: Causal models and theories, a review. Vegetation, 110, 115-147.

Motalib, A. \& Rida, A. (1994). Les vers de terre y l'énvironement. La Recherche, vol. 25.

OCP Ecuador S.A. (2001). Estudios ambientales, Linea base, capítulo 3.3 Caracterización de los Recursos faunísticos. Mayo 2001.

OCP Ecuador S.A. (2005). Estudios Complementarios - Plan de Manejo Ambiental Actividades constructivas durante el mantenimiento del DDV. Agosto 2005

Odum, E.P. (1969). The strategy of ecosystem development. Science, 164(3877), 262-270.

Robinson, G.R., Holt, R.D., Gaines, M.S., Hamburg, S.P., Johnson, M.L., Fitch, H.S., Martinko, E.A. (1992). Diverse and contrasting effects of habitat fragmentation, Science, 257 (5069), 524-526.

U.S. Department of Agriculture (USDA). (1988). Soil Taxonomy: a basic system of soil classification for making and interpreting soil surveys. USDA Soil Conservation.

Uhl, C., Nepstad, D., Silva, J.M.C. \& Vieira, I.H. (1991). Restauração de floresta em pastagens degradados, Ciencia Hoje, 13: 23-31.

Uhl, C., Nepstad, D., Buschbacher, R., Clark, K., Kauffman, B. \& Scott, S. (1990). Studies do Ecosystem Response to Natural and Anthropogenic Disturbances Provide Guidelines for Designing Sustainable Land-Use System in Amazonia. En Anderson, A. (ed.) Alternatives to deforestation; Steps toward sustainable use of the Amazon rain forest. Columbia University, Press New York, pp. 24-42. 